\title{
ANÁLISIS DE LA UTILIZACIÓN DEL RECURSO SUELO EN NICARAGUA
}

\section{Elgin Antonio Vivas-Viachica}

PhD. Facultad de Desarrollo Rural (FDR). Universidad Nacional Agraria (UNA)

Teléfono: 2233-1968, Correo Electrónico:elgin.vivas@una.edu.ni

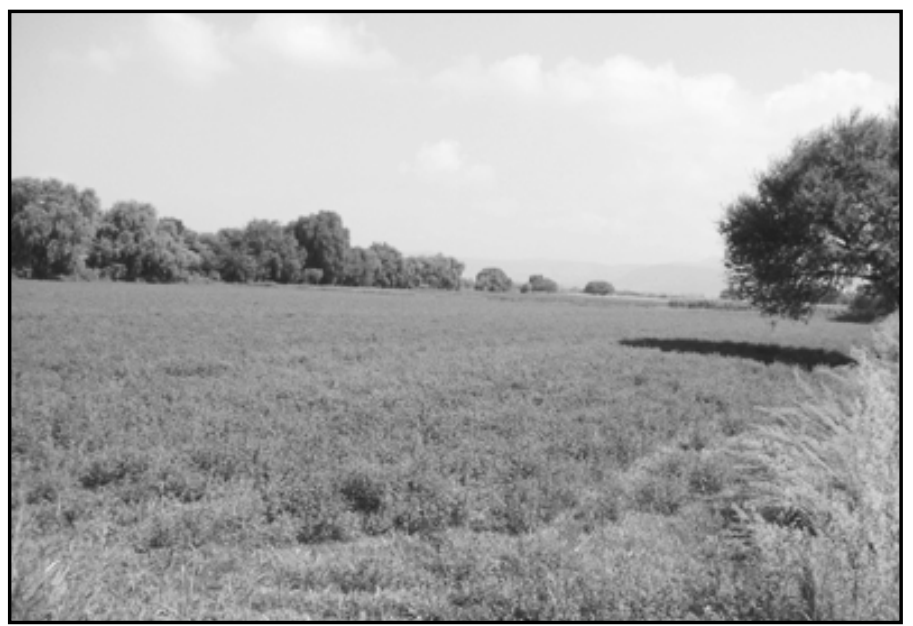

\section{RESUMEN}

El suelo se puede valorar desde una perspectiva productiva, económica, social, organizativa e histórica. En este caso se quiere hacer una valoración desde el punto de vista del suelo como mercancía, por ser objeto de compra venta. Pero hay particularidades que lo diferencian de otras mercancías, tales como no ser el resultado del trabajo precedente del hombre y tener un espacio geográfico limitado. En Centroamérica, Nicaragua es el país que tiene la más alta disponibilidad de suelo por habitante y El Salvador el que tiene la más baja disponibilidad de suelo por persona, en caso de relacionarlo con la superficie total. Pero cuando se relaciona con la superficie arable, la situación cambia para El Salvador, ya que Costa Rica tiene menos disponibilidad de suelo arable por persona. Lo cual es una buena noticia, debido a que el país dispone de más tiempo para mejorar los indicadores: productivos, ambientales, económicos y sociales. Nicaragua tiene el $82.2 \%$ del suelo de uso agropecuario, pero, la estructura de uso del área agropecuaria, se encuentra un uso extensivo. El 39.9\% es de pasto natural y el $23.1 \%$ de suelo en descanso y tacotales. Este es un ángulo de importancia, debido a la creciente necesidad de alimentos y conservación de los recursos naturales. Tomando en cuenta la cantidad de explotaciones y el área, el coeficiente de Gini es de 0.72 , el cual representa un alto nivel de concentración del uso del suelo. De acuerdo al modelo de regresión simple se ha utilizado en base a los datos de la FAO, se espera que cada año se pierdan 91760 hectáreas de bosques. De 1925000 hectáreas de tierras arables que tiene Nicaragua, lo que significa que un 4.8\% del suelo arable de nuestro país se deforesta cada año. Este es un panorama grave que se le debe prestar atención.

Palabras clave: Capa arable, uso de suelo, disponibilidad de suelo, pérdida de suelo.

\section{ABSTRACT}

We can assess the land from different perspectives: productive, economic, social, organizational and historical. In this case we want to assess soil as merchandise, because it is an object that we can sell and buy. But there are particularities differentiate that it from the other goods, such as: it is not been from the preceding work of the human beings and has a limited geographic space. In Central America, Nicaragua is the one that the highest land availability per person whichever El Salvador has the lowest land availability per person, in case of relating with the total area. But when it is related to the arable surface, the situation is different for El Salvador, since Costa Rica has less arable area availability per person. This is the good news, because the indicating country has more time to improvement: productive, environmental, economic and social situation. Nicaragua has $82 \%$ of its soil managed with plants and animals, $16 \%$ of the land of farming use, but, the structure of use of the farming area is an extensive use. The $39.9 \%$ of the remainder area grow nature pasture and $23.1 \%$ of the land remain in follow. This it is an important point of view, due to the increasing necessity of foods and conservation of the natural resources The coefficient of Gini is of 0,72 , which represents a high level of concentration of the use of the land. According to the model of simple regression that we have used on the basis of the data of the FAO, expected that every year are lost 91, 760 hectare of forests. Of 1, 925, 000 of arable area that Nicaragua has, which means that $4,77 \%$ of the arable area of our country every year is deforested. This is a serious panorama that is needed to put attention. 
$\mathrm{G}$ eneralmente, en los documentos leemos el concepto de tierra para referirnos al suelo como medio de producción. Los diferentes conceptos existentes sobre el suelo lo caracterizan como un recurso y/o medio, en el cual las plantas encuentran condiciones para desarrollar sus procesos fisiológicos. Por consiguiente, el suelo es uno de los principales recursos naturales que presenta las condiciones físicas, biológicas y químicas para que los cultivos desarrollen sus etapas fisiológicas y la respectiva producción.

El suelo en las diferentes esferas de la actividad productiva tiene diferentes significados. Por ejemplo, en la industria solamente sirve como un lugar de distribución, mientras que en la agricultura es objeto de trabajo, medio de trabajo y por ende medio de producción. Por eso siempre tiene un rol que jugar, sabiendo que no podemos producir más suelo del que se tiene. Este hecho exige que el suelo se valore desde una perspectiva productiva, económica, social, organizativa e histórica. En este caso queremos hacer una valoración desde el punto de vista del suelo como mercancía, por ser objeto de compra venta. Hay particularidades que diferencian al suelo de las otras mercancías, estas son: y calidad, a través de un impuesto. Todos estos modelos tienen su origen en esta particularidad.

Espacio geográfico delimitado. Es una particularidad de gran actualidad debido a que en realidad, la extensión del suelo por persona cada vez es menor, debido al aumento de la densidad poblacional. Cada productor debe maximizar la producción por unidad de área para poder satisfacer las crecientes necesidades de alimentos. En este caso, esta mercancía no puede reaccionar a la oferta y demanda en cuanto a aumentar o disminuir su oferta, ya que no podemos producir más suelo.

Fondo de tierra en Nicaragua, composición y estructura. Nicaragua tiene grandes recursos en extensiones de tierra, ya que posee un territorio de 13,037,347 hectáreas. Con una superficie de tierra firme de 12,034,000 hectáreas y 1,003,347 hectáreas de lagos y lagunas. Podemos decir que la densidad poblacional de Nicaragua es baja en relación a los países de la región.

Tabla 1. Disponibilidad de suelo por habitante de los países centroamericanos, 2005.

\begin{tabular}{lcr}
\hline Países & $\begin{array}{l}\text { Disponibilidad de } \\
\text { suelo (área total } \\
\text { por habitante ha }\end{array}$ & $\begin{array}{l}\text { Disponibilidad } \\
\text { (área arable po }\end{array}$ \\
\hline Guatemala & 8.41 & 0.11 \\
Honduras & 15.31 & 0.15 \\
El Salvador & 3.02 & 0.10 \\
Nicaragua & 20.85 & 0.33 \\
Costa Rica & 11.82 & 0.05
\end{tabular}

Fuente: Cálculos hechos con datos del SIECA y FAO.

No es resultado del trabajo precedente del hombre. En el caso del suelo es un regalo de la naturaleza, producto de muchos siglos de desarrollo socio-histórico, económico y natural. Todas las otras mercancías son producto del conocimiento, la inversión y el trabajo del hombre, por lo que la propiedad es indiscutible. Este hecho ha originado diferentes modelos y concepciones filosóficas que determinan su administración y uso como medio de producción y mercancía. Hay países en los cuales el suelo es propiedad social, países, en los que el suelo es objeto de compraventa sin ningún tipo de regulación y otros en los que el suelo es objeto de compraventa con ciertas regulaciones; en cuanto al manejo de la cantidad
El cuadro 1, muestra que Nicaragua tiene la más alta disponibilidad de suelo por habitante; y El Salvador el que tiene la más baja disponibilidad de suelo de área por persona, en caso de relacionarlo con la superficie total. Pero cuando se relaciona con la superficie arable, la situación cambia para El Salvador, ya que Costa Rica tiene menos disponibilidad de suelo arable por habitante.

Podemos observar en estos análisis que se interrelacionan dos importantes variables: población y recurso suelo. Sabemos que de acuerdo a los datos censales del INIDE la tasa de crecimiento poblacional de Nicaragua disminuyó en el período intercensal 1971- 1995 de 3,5\% a $1,7 \%$ en el período intercensal 1995- 2005, lo cual es una buena noticia, debido a que el país dispone de 
más tiempo para mejorar sus indicadores: productivos, ambientales, económicos y sociales.

Un primer panorama sobre el uso del suelo, son los resultados del Censo Agropecuario 2003, presentados por INIDE. En los que se puede ver que el peso porcentual de las explotaciones aumenta sistemáticamente desde propiedades con menos de cinco hectáreas hasta propiedades con 50 hectáreas y después comienzan a descender, pero en el caso de la superficie utilizada por estas propiedades no desciende, lo cual es concentración del uso del suelo, y desde luego que tiene sus implicaciones económicas y sociales. Esta realidad presupone el diseño de políticas que mejoren la eficiencia de uso del suelo.
Ahora bien, este panorama general del país es el punto de partida para realizar un análisis exhaustivo del uso del suelo, tal a como está presentado en el cuadro No. 3 que refleja el comportamiento del uso del suelo. Observando, el 82.16\% del suelo es agropecuario, pero la estructura de uso del área agropecuaria, se encuentra un uso extensivo del suelo. El 39.94\% es de pasto natural y el $23.12 \%$ de suelo en descanso y tacotales. Este es un ángulo de importancia, debido a la creciente necesidad de alimentos y conservación de los recursos naturales.

Profundizando un poco más para ver la distribución del suelo, se grafica la curva de Lorenz. Sabiendo que un aspecto importante para que el suelo, como mercancía, tenga efecto multiplicador en la producción de alimen-

Tabla 2. Distribución del uso del suelo por tamaño de las explotaciones en Nicaragua

\begin{tabular}{lccrr}
\hline $\begin{array}{l}\text { Tamaño de las } \\
\text { explotaciones }\end{array}$ & $\begin{array}{l}\text { Total de las } \\
\text { explotaciones }\end{array}$ & $\begin{array}{l}\text { Porcentaje de } \\
\text { explotación }\end{array}$ & \multicolumn{1}{l}{$\begin{array}{l}\text { Superficie } \\
\text { en ha }\end{array}$} & Porcentaje \\
\hline 0.5 a menos & 7337 & 3.68 & 1949 & 0.03 \\
0.51 a 1 & 10745 & 5.38 & 7197 & 0.11 \\
1.01 a 2.5 & 21379 & 10.71 & 28592 & 0.45 \\
2.51 a 5 & 26517 & 13.29 & 73328 & 1.16 \\
5.01 a 10 & 28576 & 14.32 & 160438 & 2.55 \\
10.01 a 20 & 27022 & 13.54 & 300851 & 4.78 \\
20.01 a 50 & 38780 & 19.43 & 989324 & 15.71 \\
50.01 a 100 & 21684 & 10.87 & 1180798 & 18.75 \\
100.01 a 200 & 10746 & 5.39 & 1148139 & 18.23 \\
200.01 a 500 & 5169 & 2.59 & 1161266 & 18.44 \\
500.01 a más & 1594 & 0.80 & 1247308 & 19.80 \\
\hline Total & 199549 & 100.00 & 6299189 & 100.00
\end{tabular}

CENAGRO, 2003.

Tabla 3. Uso actual del suelo en Nicaragua

\begin{tabular}{|c|c|c|c|}
\hline Cultivos anuales o temporales & 675 & & 13.04 \\
\hline Cultivos permanentes y semi-permanentes & 298029 & & 5.76 \\
\hline Tierras en descanso (tacotales) & 1196385 & & 23.12 \\
\hline Pastos naturales & 2067288 & & 39.94 \\
\hline Pastos cultivados o sembrados & 938640 & & 18.14 \\
\hline Subtotal agropecuaria & 5175448 & 82.16 & 100.00 \\
\hline Bosques & 895626 & 14.22 & \\
\hline Instalaciones y viales & 71187 & 1.13 & \\
\hline Pantanos, pedregales, otras tierras & 113176 & 1.8 & \\
\hline Tierras afectadas por fenómenos naturales & 43754 & 0.69 & \\
\hline Superficie total & 6299189 & 100.00 & \\
\hline
\end{tabular}

Fuente: CENAGRO, 2003. 
tos y la exportación: es la distribución de este medio de producción.

La curva de Lorenz permite ver las inequidades en la de las exportaciones, la oferta en el mercado local y la disminución en la generación de empleo del sector rural.

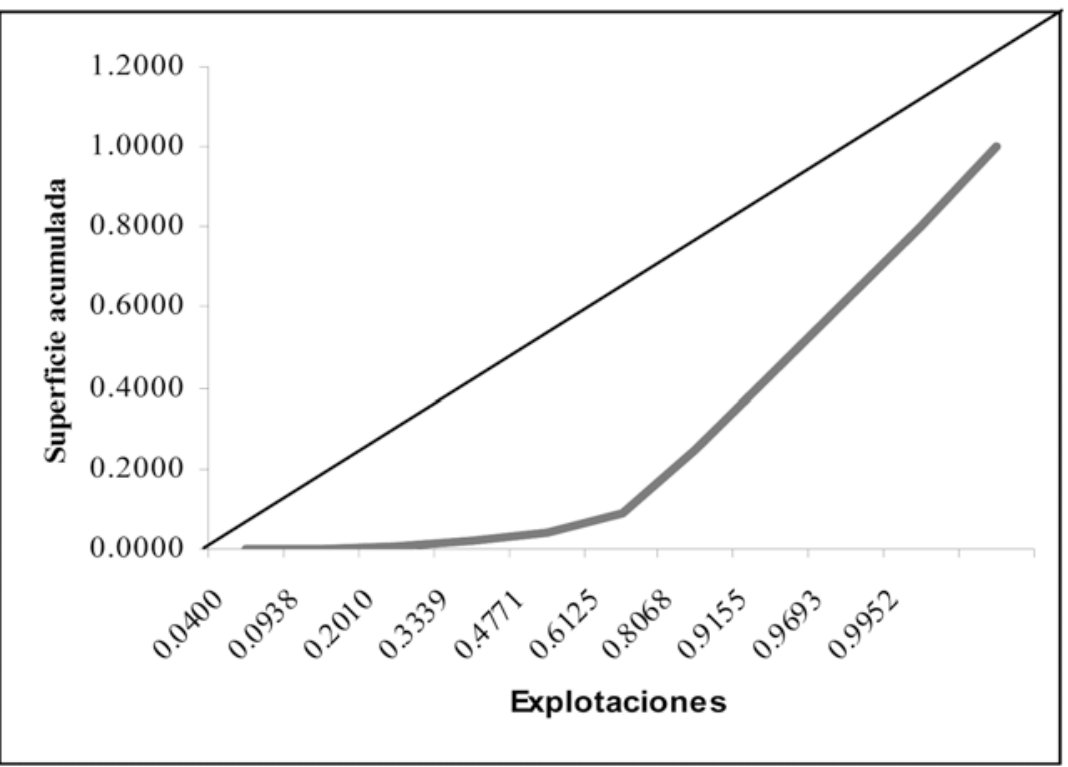

Figura 1. Curva de Lorenz, fuente: Elaboración propia en base a datos de CENAGRO.

distribución del suelo. En este caso en el eje X están las cantidades acumuladas de explotaciones agropecuarias y en el eje Y la superficie acumulada. De manera que el área ubicada entre la curva y la recta, expresa la inequidad en la distribución de la tierra. Entre mayor es el área mayor es la inequidad. Nicaragua tiene alta inequidad en la distribución del uso del suelo. Lo que es un factor importante en la falta de diversificación
Tomando en cuenta el número de puntos calculados en la curva de Lorenz, se calcula el coeficiente de Gini, a traves de la siguiente fórmula:

$$
\mathrm{G}=1-\sum_{\mathrm{n}=1}^{\mathrm{n}=\mathrm{i}}(\mathrm{Yn}+\mathrm{Yn}-1) *(\mathrm{Xn}-\mathrm{Xn}-1)
$$

Tabla 4. Cálculo de coeficiente de Gini

\begin{tabular}{ccccccc}
$\begin{array}{c}\text { Estructura de la } \\
\text { superficie }\end{array}$ & $\begin{array}{c}\text { Aculado de la } \\
\text { superficie }\end{array}$ & Yn+Yn-1 & $\begin{array}{c}\text { Estructura de las } \\
\text { explotaciones }\end{array}$ & $\begin{array}{c}\text { Acumulado } \\
\text { de las } \\
\text { explotaciones }\end{array}$ & Xn-Xn-1 & Y*X \\
\hline & & & & & \\
\hline .00 & 0.00 & 0.00 & 0.04 & 0.01 & 0.01 & 0.0 \\
0.00 & 0.00 & 0.00 & 0.05 & 0.07 & 0.05 & 0.0 \\
0.00 & 0.01 & 0.01 & 0.11 & 0.17 & 0.11 & 0.0 \\
0.01 & 0.02 & 0.02 & 0.13 & 0.31 & 0.13 & 0.0 \\
0.03 & 0.04 & 0.06 & 0.14 & 0.45 & 0.14 & 0.01 \\
0.05 & 0.09 & 0.13 & 0.14 & 0.59 & 0.14 & 0.02 \\
0.16 & 0.25 & 0.34 & 0.19 & 0.78 & 0.19 & 0.07 \\
0.19 & 0.44 & 0.68 & 0.11 & 0.89 & 0.11 & 0.07 \\
0.18 & 0.62 & 1.05 & 0.05 & 0.94 & 0.05 & 0.06 \\
0.18 & 0.80 & 1.42 & 0.03 & 0.97 & 0.03 & 0.04 \\
0.20 & 1.00 & 1.80 & 0.01 & 0.98 & 0.01 & 0.01 \\
1.00 & & & 1.00 & & & 0.28 \\
\hline G & & & & & 0.72 \\
\hline
\end{tabular}


El coeficiente de Gini mide las desigualdades, en este caso con los datos del cuadro No.2, donde hay datos de la cantidad de explotaciones y el área que tienen, ordenada por concentración de la superficie utilizada.

La expresión matemática de la curva de Lorenz es el coeficiente de Gini, que en este caso es de 0.72, el cual representa un alto nivel de concentración del uso del suelo.
Es decir, el mejoramiento de la estructura de siembra puede darse tomando el criterio, solamente, de la apertura que tengan determinados productos en el mercado nacional e internacional. Para garantizar la sostenibilidad de la producción tienen que tomarse en cuenta los tres criterios, antes mencionados: Comercialización, Tecnología y Cultura agrícola de los productores. Debe de hacerse un esfuerzo por mejorar la distribución del uso del suelo. Impulsando políticas, estrategias y programas que ayuden

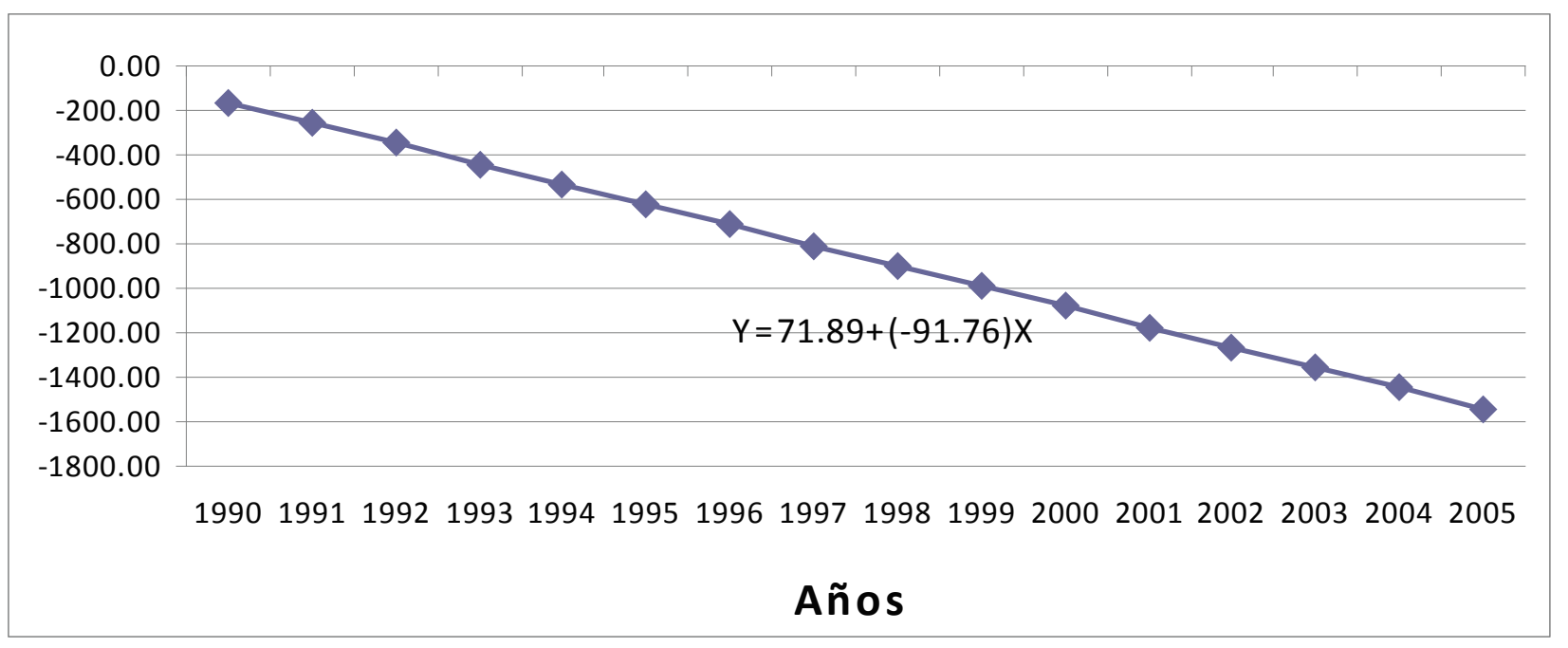

Figura 2. Curva de regresión de la deforestación en Nicaragua en miles de hectáreas, Fuente: Elaboración propia en base a datos de la FAO.

Además, hay que agregar factores de la conservación del suelo.

Un aspecto clave que hay que analizar es el nivel de deforestación, lo cual tiene que ver con la pérdida de la calidad del suelo y el avance de la frontera agrícola. De acuerdo al modelo de regresión simple que hemos utilizado en base a los datos de la FAO, se espera que cada año se pierdan 91,760 hectárea de bosques. De 1,925,000 hectáreas de tierras arables que tiene Nicaragua, lo que significa que un $4.77 \%$ del suelo arable de nuestro país se deforesta cada año. Se tiene un panorama agudo que se le debe prestar atención.

Principales estrategias para la utilización efectiva y conservación del recurso suelo. Para un país pobre como Nicaragua el recurso suelo adquiere una importancia vital como mercancía dinamizadora de la economía, pero, al mismo tiempo es un recurso natural. Por lo que se requieren políticas que disminuyan esta contradicción aparente.

Hay que mejorar la estructura de las áreas de siembra, a través de factores de comercialización, tecnologías, conocimientos e información que los productores tengan. a un pensamiento de producción intensiva y racional.

El principal camino para el incremento de la efectividad económica de la utilización del suelo en la agricultura, es la intensificación. Que tome en cuenta, tanto, tecnología local y de punta. Evitando una mentalidad de producción extensiva e irracional, que provoca una necesidad de tener áreas extensa de suelo.

Es importante cambiar la tendencia que se observa en la curva de regresión de la deforestación, para mantener la cubierta vegetal, los ecosistemas y calidad de los suelos y disminuir la desertificación. Pensando en el mejoramiento del suelo utilizable y del aprovechamiento de las distintas clases de suelo comprendidas en la explotación agrícola; incrementar la apertura al cultivo de suelos ociosos y el mejoramiento del suelo arable.

El hombre es central en el proceso de producción, de manera que sus conocimientos, destrezas, habilidades son factores esenciales para un uso sostenido del recurso suelo como principal medio de producción en la agricultura. Sabiendo que los alimentos dependen de la actividad agrícola y la demanda de alimentos se incrementa año con año. 


\section{REFERENCIAS BIBLIOGRÁFICAS}

Arnon, I. 1987. La modernización de la agricultura en países en vías de desarrollo: Recursos potencialesproblemas. Editorial Limusa, 733pp-

Buol, SW; Hole, FD; Mc Cracken, RJ. 1991. Génesis y clasificación de suelos, 2da. edición, México, editorial Trillas, , 417 pp.

FAO. 1981. Agricultura Horizonte 2000. Roma,. pág. 41-63.

FAO. 2008. Datos sobre la tierra en http://faostat.fao.org/site/377/default.aspx, accesado el 24 de Julio del 2008.

Fitz Patatrick EA. 1987. Suelos. Su formación, clasificación y distribución, editorial continental, México tercera reimpresión , 430 pp.

Food And Agriculture Organization Of The United Nations. 2001. Rome,vol.53, Statiscs series No. 156.

Foster, AB. 1990. Métodos aprobados en conservación de suelos, Editorial Trillas, Sexta reimpresión, 411 pp.

Fuentes Yagüe, JL. 1989. El suelo y los fertilizantes. Tercera edición revisada y ampliada, Editorial Mundi Prensa, España, 1989, 283 pp.

INETER. 2008. Caracterización geográfica del territorio nacional, en http://www.ineter.gob.ni/ caracterizaciongeografica/capitulo6.html, acceso el 25 de junio del 2008.

Rodríguez Morales, R. 1981. Prácticas de conservación de suelos, Editorial Pueblo y Educación, 135 pp.

Schawab, GO. 1990. Ingeniería de conservación de suelos y aguas, editorial Limusa, Primera edición, 571 pp.

SIECA. 2008. Centroamerica: población estimada total, por año calendario, según sexo,19952005.Enhttp:// www.sieca.org.gt/Publico/CA_en_cifras/Serie_33/Poblacion/001_PobTot\%20datos\%20enero\%202003_ web.htm.

Villanueva Ortiz, B. 1977. Fertilidad de suelos, Chapingo, México, 209 pp. 